\title{
Variations
}

Variations

Revue internationale de théorie critique

15 | 2011

La haine

\section{La haine du prolétariat par les classes dominantes}

José Chatroussat

\section{(2) OpenEdition}

Journals

Édition électronique

URL : http://journals.openedition.org/variations/97

DOI : 10.4000/variations.97

ISSN : 1968-3960

\section{Éditeur}

Les amis de Variations

\section{Édition imprimée}

Date de publication : 1 mars 2011

\section{Référence électronique}

José Chatroussat, "La haine du prolétariat par les classes dominantes », Variations [En ligne],

15 | 2011, mis en ligne le 01 février 2012, consulté le 01 mai 2019. URL : http://

journals.openedition.org/variations/97 ; DOI : 10.4000/variations.97 
José Chatroussat

La haine du prolétariat par les classes dominantes

Comment s'exprime la haine des classes dominantes à l'égard du prolétariat et plus généralement des classes populaires ? Existe-t-elle encore aujourd'hui ? Et si oui quel rôle joue-telle dans les rapports sociaux actuels?

Il peut sembler au premier abord que les capitalistes, détenant tous les pouvoirs essentiels et tirant en quelque sorte les principales ficelles du jeu politique et social à l'échelle mondiale, n'ont pas besoin d'exprimer leur haine des prolétaires ni même de l'éprouver tant leur position présente semble inébranlable et indépassable si on n'accorde pas une importance exagérée au lot inévitable des grèves sporadiques, des émeutes populaires et coups de colère en apparence sans lendemain. L'indifférence ou le mépris devrait suffire.

Et pourtant il n'en est rien. La crise sous sa forme financière qui a éclaté au cours de l'été 2007 pour prendre une forme spectaculairement explosive en septembre 2008, a ébranlé cette posture narquoise et autosatisfaite qui avait prévalu pendant les années qui ont suivi immédiatement la chute du mur de Berlin en 1989. « L'empire du mal » était vaincu par épuisement de l'adversaire et ralliement enthousiaste des dictateurs bureaucrates aux délices très lucratifs de l'économie de marché. La caste dirigeante en Chine devait significativement s'ouvrir la voie de grands succès au sein de l'économie capitaliste mondiale en procédant, en cette même année 1989, à une répression sanglante à la fois à Pékin place Tienanmen et dans plusieurs grandes villes contre le mouvement d'émancipation lancé par les étudiants et rejoint par de jeunes ouvriers. 
Depuis plusieurs décennies la menace prolétarienne bien vivante de 1917 à 1937 s'était comme chosifiée en «bloc de l'Est» où, au demeurant, les dictateurs locaux savaient mater les ouvriers y compris de façon sanglante comme on l'avait vu en 1953 à Berlin Est, en 1956 en Hongrie et en 1970 en Pologne pour ne citer que les mouvements et les répressions les plus importantes. Tout cela à la plus grande satisfaction des bourgeoisies qui avaient déjà fort à faire dans leur sphère pour tenter d'écraser les mouvements d'émancipation des peuples en Asie, en Afrique et en Amérique Latine.

Ce fameux bloc nommé abusivement soviétique était comme un ensemble de collines morainiques, le témoignage de la progression d'un grand glacier ayant disparu, à savoir la vague révolutionnaire qui s'est levée en Russie en février 1917 et s'est prolongée en Allemagne à partir de novembre 1918. Témoignage à la fois déplaisant et bien utile car il servait de faux horizon historique pour les peuples et les exploités rêvant de s'émanciper du joug impérialiste.

Il ne reste plus depuis vingt ans qu'un seul « bloc », le bloc capitaliste dont la configuration géographique couvre la totalité de la planète et de son environnement, avec ses failles et ses rivalités «multipolaires » comme disent les géopoliticiens. Pour parvenir à ce résultat qui se présente surtout comme une impasse pour l'humanité, la haine des classes dominantes à l'égard des « classes dangereuses » comme on les qualifiait au XIXe siècle aura joué un rôle décisif.

\section{La haine des classes dominantes dans son expression directe}

Le comportement des bourgeois à l'égard de leur serviteurs, employés, ouvriers et autres salariés qu'ils nomment volontiers « leur personnel » reste dans l'ensemble serein tant qu'ils restent « à leur place », c'est-à-dire à leur poste et au travail pour le compte de leur employeur. Mais ils se voient très spontanément qualifiés d'irresponsables, de saboteurs de l'économie nationale, de 
« preneurs d'otages du public », dès qu'ils quittent leur place assignée. Ceux qui n’ont pas de travail sont invités à raser les murs ou à les tenir, sinon ils se voient qualifiés de « racaille ».

Tant que les travailleurs contribuent sans embûches sans rechigner à la reproduction du capital dans son ensemble, ils ne sont que du capital variable. Ils existent sous la dénomination de « charges salariales » dont il faut s'efforcer de diminuer le coût ou de « ressources humaines » qu'il faut gérer de façon optimale. Dans cet état quasi chosifié, les prolétaires ne provoquent aucun affect agressif de la part des patrons et actionnaires qui du reste n'ont ni le besoin ni l'envie d'être en contact avec eux. Ils s'efforcent de les ignorer le plus possible et de ne même pas leur répondre en tant que personnes. Quand un plan de licenciements est annoncé dans une entreprise, la formule rituelle à l'adresse des médias est toujours la même : «La direction n'a pas souhaité s'exprimer. »

L'hypothèse qu'il restera à vérifier en ce qui concerne notre époque est que la haine des classes dominantes prend toujours, comme dans le passé, une expression directe, compulsive dès que des gens des classes populaires ont la prétention inouïe d'occuper l'espace public avec leurs propres désirs et revendications. Une rapide plongée dans le passé permettra d'apprécier le caractère répétitif de ce mécanisme tout à la fois psychologique, social et politique.

Dans le cas des révoltes des Canuts lyonnais en 1831 et en 1834, de l'insurrection des travailleurs parisiens en juin 1848 et de la Commune de Paris de mars à mai 1871, l'espace est occupé et défendu les armes à la main par des hommes et des femmes qui ont déserté leur poste d'exploités. La circonstance la plus aggravante est l'affirmation par ces nouveaux acteurs qui ont surgi sur la scène de l'histoire, d'être des pionniers dans la construction d'un monde meilleur, d'une autre société débarrassée des classes dominantes. Leur haine en réponse est alors une passion de destruction pure et simple des insurgés porteurs d'espoir et de laminage idéologique et calomniateur de longue haleine pour défigurer la teneur de ces épisodes. 
Il faut insister sur un point qui concerne aussi bien les insurrections des Canuts de Lyon en 1831 et 1834, l'expérience de la Commune de Paris au printemps 1871, la grève des salariés occupant leurs lieux de travail en juin 1936 en France, la révolution Espagnole de 1936-39, le mouvement de Mai 68, celui des travailleurs et étudiants de Cordoba en Argentine en mai 1969, le mouvement place Tienanmen en 1989, le mouvement zapatiste au Chiapas ou encore celui d'Oaxaca à l'été 2006 : dans l'ensemble tout se passe bien dans l'espace contrôlé par les acteurs de ces luttes. Le scandale absolu pour les riches et leurs hommes d'État, c'est que la société fonctionne mieux lorsqu'ils sont court-circuités, paralysés, mis momentanément de côté. Cela s'exprime par une bonne humeur générale et une baisse très significative de la délinquance.

S'il ne s'agissait que de velléités de bâtir un monde futur, passe encore ! Mais l'expérience du bonheur individuel au sein d'une collectivité euphorisée par ses capacités mise en oeuvre, loin de la pression asphyxiante de l'exploitation, loin des institutions bureaucratiques et étouffantes, une telle expérience relève du vécu et non d'une vue de l'esprit facile à moquer, à dénigrer ou à plonger dans l'oubli.

On a vu de façon récurrente que la volonté des gens d'intervenir dans le champ sacré de la politique «sérieuse » de façon autonome, même sur un point particulier n'affectant pas le fonctionnement d'ensemble de la société, déclenche des campagnes idéologiques étonnamment agressives. Nous en avons eu des exemples significatifs à notre époque. Il y a eu la campagne médiatique hystérique visant à faire voter oui au Traité Constitutionnel Européen. Bien des commentateurs ne cachaient pas leur regret qu'on ait demandé leur avis par un vote à un peuple composé d'individus aussi obtus. La rage contre les masses qui ne comprennent rien aux « enjeux » faisait bon ménage avec la rage contre la démocratie.

Il est non moins significatifs que la révolte des jeunes de banlieue ait été systématiquement 
pensée comme le fait d'individus insensés, agissant sans autre motivation que le plaisir de détruire, alors que bon nombre d'entre eux avaient formulé dès le début, de façon extrêmement claire et ramassée, une revendication politique : «Sarkozy (alors ministre de l'Intérieur) doit s'excuser» ou « il doit démissionner ». On décréta qu'ils n'avaient rien dit, qu'ils étaient incapables de verbaliser une revendication.

Sans reprendre les éléments de cette crise, il faut rappeler cependant que le gouvernement ne s'en est pas tenu à une répression policière et judiciaire massive. Là encore ce qui reste de balisage démocratique fragile devait être mis au rancart. Fouetté par la peur et dans une perspective de préparation des esprits aux guerres civiles à venir, il décréta « l'état d'urgence » avec la complicité ou le silence embarrassé de toute la gauche. Les molles protestations de l'extrême gauche lui permirent de sortir de son silence et de l'état de sidération dans lequel cette révolte l'avait mise. Peu d'intellectuels avaient réussi à s'affranchir du «climat» ambiant et dominant qui parvint à stigmatiser durablement les jeunes des banlieues populaires.

\section{Un grand écrivain face à « messieurs les ouvriers »}

La fonction d'écrivain, de philosophe ou d'artiste n'offre aucune garantie en elle-même face à la prégnance d'une passion destructrice se répandant dans un corps social. De même que la personnalité autoritaire existe dans toutes les classes sociales, la haine sociale n'est pas l'apanage ni d'une minorité d'hommes d'Etat et de patrons arrogants et hystériques, ni de gens des milieux populaires frustes et dépourvus de culture.

C'est pour approcher de la compréhension des sources individuelles et sociales de la haine qu'il sera question à présent d'un cas plus complexe et plus troublant, celui de Gustave Flaubert. Il s'agit d'une personne hautement cultivée qui n'était pas directement impliquée dans la mise au 
travail de salariés. Son statut social est celui d'un écrivain indépendant, non conventionnel, cherchant bien difficilement à vendre ses écrits.

Le récit de Flaubert intitulé Un Cœur simple publié en 1876 commence par cette phrase : «Pendant un demi-siècle, les bourgeoises de Pont-L'Evêque envièrent à Mme Aubain sa servante Félicité. » En effet elle sait tout faire et travaille sans relâche pour une rémunération annuelle très modeste. Elle « resta fidèle à sa maîtresse qui cependant n'était pas une personne agréable. »

L'écrivain n'a aucun mépris, aucune condescendance pour cette travailleuse. Au contraire. Il nous la rend attachante dans sa quête d'affection toujours déçue ou trahie à l'égard de son neveu, des enfants de sa maîtresse ou d'autres personnes. L'évolution fantasque de Félicité qui reporte sa quête d'amour sur un perroquet n'appelle pas la moquerie. Certes elle est restée à sa place aliénée de servante. Mais elle a cherché à sublimer sa condition dans un objet d'amour imaginaire. Flaubert est fasciné par toutes les tentatives de sublimation, même si elles sont vouées à l'échec ou au délire mystique, pour échapper au monde terre à terre des bourgeois calculateurs et des positivistes qui ont tout compris.

En tant que romancier moderne, Flaubert, indépendamment de ses opinions dans la vie, se conduit en vrai démocrate à l'égard de ses personnages et notamment celui de Félicité. Il n'a pas à cautionner les préjugés et les hiérarchies sociales. En créateur mobilisant son talent et sa marge de liberté, il peut, comme son jeune ami Maupassant, critiquer avec acuité la médiocrité et l'hypocrisie des bourgeois.

Cinq ans plus tôt, en 1871, Gustave Flaubert avait fait chorus avec la plupart des écrivains, artistes et journalistes de renom pour exprimer sa haine sans bornes des Communards. Il avait réagi en bourgeois ordinaire, terrorisé par les ouvriers et réclamant la terreur à leur égard. On lit dans sa correspondance : « Je trouve qu'on aurait dîu condamner aux galères toute la Commune et forcer 
ces sanglants imbéciles à déblayer les ruines de Paris, la chaîne au cou et en simples forçats. » Il écrit à George Sand : «Ah! Dieu merci, les Prussiens sont là, c'est le cri universel des bourgeois. Je mets dans le même sac messieurs les ouvriers, et qu'on $f$...le tout ensemble dans la rivière !... »

Ces artisans, ouvriers, employés et gens de maison n'étaient pas restés « à leur place ». Ils et elles étaient partis ensemble «à l'assaut du ciel». L'espace de Paris avait été occupé, géré, gouverné, transfiguré par ces gens-là, autant dire profané ! La haine des bourgeois à l'égard des prolétaires jailli à gros bouillons. Les massacres et la répression durable la plus brutale et la plus arbitraire à l'égard des Communards se trouvent pleinement justifiées par « l'élite de la culture française » en proportion de la terreur qu'elle a éprouvée devant cette expérience prolétarienne, menace palpable à l'égard de leurs fonctions, de leurs propriétés et de leur image d'élite irremplaçable.

La puissance de la rage était telle que Victor Hugo, gloire littéraire nationale s'il en fut, a été traîné dans la boue par toute la presse versaillaise, non pas pour avoir soutenu la Commune, mais pour s'être indigné des excès de la répression. Victor Hugo échappe à la haine vulgaire dominante et se désole de la déchirure dans le corps social de la nation française.

L'exemple de Flaubert montre que le comportement d'un individu en phase avec les réactions à chaud des Versaillais n'implique pas une aversion systématique pour les gens issus des classes populaires mais un refus non négociable de les voir occuper une autre position dans la société que celle qui leur est fortement assignée : être un prolétaire soumis, devenir un bourgeois, mais en aucune manière être partie prenante d'un collectif d'hommes et de femmes voulant ouvrir une voie vers l'émancipation.

Pour «mobiliser» la haine de Flaubert comme de bien d'autres, il a fallu qu'il se reconnaisse dans des valeurs et des institutions préexistantes, la République (celle des nantis apeurés basée à Versailles), la France, la propriété... A ce point de notre enquête, l'approche 
psychanalytique devient indispensable. Flaubert n'avait aucun intérêt personnel à haïr les ouvriers parisiens. Après tout, les détériorations à Paris où il avait plaisir à se retrouver avec ses amis étaient principalement le fait de l'armée des Versaillais. Alors ?

\section{Les racines de la haine}

Pour prendre un exemple contemporain, Alain Finkielkraut, le professeur de philosophie à l'École polytechnique et producteur d'une émission sur France Culture, n'a aucun intérêt personnel à haïr les jeunes des banlieues populaires, tout particulièrement ceux d'origine maghrébine. Ils n’ont pas brûlé sa voiture ni perturbé ses cours. Dans le cas de Flaubert comme de Finkielkraut, la haine passe par des représentations constituées de la Culture (occidentale), la Nation (française), l'Autorité toujours nécessaire pour mater les débordements des bas instincts populaires.

La précieuse culture dont ils se croient à tort ou à raison les éminents détenteurs devient un argument et même un aliment pour nourrir leur haine ; ce qui ne peut manifestement pas être la préoccupation des patrons et des politiciens faisant tirer sur des grévistes ou des manifestants, que ce soit à l'époque de Flaubert ou à celle de Finkielkraut. A la fois dans la constitution de leur personnalité et dans le cadre institutionnalisé dans lequel ils évoluent et tiennent un rôle, ce qui a du sens est entièrement de leur côté, de leur Moi, et se trouve défini par des frontières symboliques à la fois sociales et subjectives au-delà desquelles on pénètre dans le domaine de l'Autre, l'Ennemi, le Barbare, le Prussien, le Communard, le Musulman (forcément fanatique) qui agit de façon insensée. Cornelius Castoriadis a donné une interprétation psychanalytique fouillée et pénétrante des racines psychiques et sociales de la haine [in Figures du pensable, Seuil, 1999]. Il n'est pas possible de reprendre tous les éléments de son argumentation qui s'appuie sur les recherches de Freud et les approfondit par leur inscription dans ce qu'il appelle l'institution imaginaire de la société. «Être socialisé signifie d'abord et surtout investir l'institution existante de la société et les significations 
imaginaires que cette institution porte. » Castoriadis distingue deux sources de la haine :

«- La tendance fondamentale de la psyché à rejeter (et ainsi, à haïr) ce qui n'est pas elle-même ;

- la quasi-nécessité de la clôture de l'institution sociale et des significations imaginaires qu'elle porte. »

Toutes les relations du sujet sont habitées par l'ambivalence de l'amour de soi et de la haine de soi (qui est perçu comme un autre). Au niveau de la psyché individuelle (qui est essentiellement une fabrication sociale), l'envers de l'investissement positif de soi est la haine de l'autre. Un sophisme puissant et élémentaire le soutient: «(Le) bien, c'est moi. Lui n'est pas moi. Donc, il n'est pas bien (ou l'est moins que moi). » S'il se trouve que « je » suis français, anglais allemand, italien, hutu, hétérosexuel, etc, l'Autre haïssable est mis à disposition des sujets par les indications langagières de telle ou telle institution sociale dans laquelle « je » évolue et s'est fabriqué. Car considère Castoriadis : «L'institution sociale est donatrice de sens. Elle répond plus ou moins bien au besoin primordial de la psyché : le besoin de sens. »

L'intérêt de cette analyse est qu'elle établit un lien entre la racine psychique et la racine sociale de la haine, ce qui à mon avis l'apparente à l'analyse de la personnalité autoritaire par Adorno et ses collaborateurs (lire à ce propos la synthèse des résultats de cette enquête dans l'introduction et le premier chapitre de Conscience de casse d'Alexander Neumann, Variations Hors-Série Hiver 2009/2010).

L'analyse psychanalytique et sociale de Castoriadis nous dégage de toute forme de réductionnisme. Elle ne noie pas les sources de la haine dans l' « essence » d'une société donnée ni dans l' « essence » intrinsèque du sujet individuel. Les processus sociaux de donation de sens qui effectuent le passage du sujet à un individu socialisé sont complexes, mouvants et multiformes.

Cette analyse met en relief que dans tous les cas de figure, les potentiels de haine sont toujours là, chez n'importe quel sujet, à des degrés variables selon les sujets et selon les situations. Ces 
potentiels sont toujours mobilisables par l'institution sociale (comme instance extérieure et intériorisée), à bas bruit ou sous une forme massive et paroxystique.

\section{L'intoxication xénophobe ou ethnocidaire par les couches dirigeantes}

Les préparatifs d'intoxication qui conduisent des peuples constitués massivement de travailleurs des villes et des campagnes à se massacrer les uns les autres ou à ce qu'une partie en massacre une autre (génocides des Arméniens, des Juifs, des Tziganes et des Tutsis) mettent en jeu ces ressorts. Ils impliquent toujours les couches dirigeantes d'une institution sociale.

Ainsi les prolétaires et les paysans français et allemands ne se sont pas rués subitement les uns contre les autres en août 1914, ils l'ont fait suite à une convocation, une mobilisation générale décrétée par leurs Etats respectifs. Si les simples soldats ne savaient pas qu'ils allaient participer à, et pour une grande part disparaître dans un massacre qui fit en un peu plus de quatre ans quinze millions de morts, les ministres, membres d'État-major, diplomates et les capitalistes les plus concernés par cette entreprise inédite de massacre industriel en étaient parfaitement conscients, comme l'attestent maintes déclarations préliminaires. Elles ont été relevées par l'historien Arno Mayer dans les dernières pages de son livre, La Persistance de l'Ancien Régime, L'Europe de 1848 à la Grande Guerre (Flammarion, 1983).

La haine était préexistante chez bien des soldats mais à des doses très variables et de toute façon pas à un niveau initial tel qu'ils aient pu s'engager durablement dans une entreprise de massacre aussi monstrueuse. A telle enseigne que le sens ultime de leurs actes était de tuer pour en finir avec une guerre censée être la dernière parce que trop énorme par sa barbarie.

Mais lorsque le mécanisme de la haine collective a réussi à être mis en route, cela marche 
tout seul comme le dit cyniquement un personnage imaginé par le philosophe Günther Anders à la fois proche et en marge du courant dit de l'École de Francfort : "La règle de la haine s'énonce comme suit : " Je combats quelqu'un - de ce fait je me mets à le hä̈r. L'appétit vient en mangeant, la haine vient en luttant. Je le hais. Là-dessus, je le combats encore plus sauvagement. Les deux, haine et combat, s'intensifient réciproquement. » [La haine à l'état d'antiquité, Rivages, 2007]

Anders pointe dans ce recueil la logique de la haine au cours de la Première et de la Seconde Guerres Mondiales qui atteint ses sommets de barbarie avec Auschwitz et Hiroshima. Il argumente également sur le fait que la guerre industrielle permet aussi de massacrer « sans haine » comme les pilotes américains lâchant des bombes et du napalm sur les villages vietnamiens.

Le vent de la haine, de la xénophobie et de la barbarie s'est le plus souvent levé en premier lieu au sein des secteurs des milieux aisés les plus cultivés et les mieux informés de la marche du monde.

Il est connu que c'est à l'appel de Radio Mille Collines et des prêtres catholiques au Rwanda en avril 1994 que la population hutu a été appelée à « faire le travail chaque jour », euphémisme signifiant tuer toute la population tutsi ainsi que les Hutu voulant les protéger ou ne voulant pas « faire le travail ».

Parce que cela n'a pas été suffisamment relevé en son temps et que cela est déjà largement oublié, il faut rappeler qui a lancé les feux de la haine ethnique qui ont mené à la dernière guerre en Europe, celle qui a conduit à l'explosion de la Yougoslavie à partir de 1991. Ils ont été provoqués délibérément par des historiens, des romanciers, des poètes, des scientifiques se découvrant une nouvelle identité nationale et dénichant dans le passé les symboles de leur grandeur nationale serbe, croate, etc. Et cela en connivence avec les castes politiciennes rivales et les affairistes qui aspiraient 
par tous les moyens à avoir leur Etat et leurs ressources propres plutôt que de faire face à des mécontentements sociaux grandissants, surtout de la part des salariés yougoslaves.

Au tout début de ces mascarades nationalistes qui préparèrent de multiples massacres, les travailleurs yougoslaves qui vivaient en France furent stupéfaits et incrédules. C'était une mauvaise blague si la guerre pouvait s'introduire entre amis de longue date ou dans leur couple et leur famille sous prétexte que les uns étaient d'origine serbe, d'autres d'origine croate, kosovar ou bosniaque ! La guerre prit un tour d'autant plus féroce et prolongé en Bosnie que dans cette partie du pays les origines et les cultures étaient particulièrement mélangées ou se côtoyaient plutôt en bonne intelligence.

\section{La sous-traitance de la haine}

Les parcours variés dans le champ de la haine de masse nous conduisent à la situation actuelle des classes dominantes confrontées aux aléas de leur économie et de leur domination.

Bien que souhaitable pour eux, la posture d'impassibilité face aux classes populaires est difficile à tenir. Les bourgeoisies nationales comme la partie transnationale des capitalistes se sont probablement persuadées que le prolétariat était historiquement vaincu, sans cohésion menaçante pour leur pouvoir. Elles peuvent traiter les grèves et les mouvements de colère populaires les uns après les autres en mettant à contribution à la fois leurs policiers et leurs juges, et les gardiens de la paix sociale que sont les appareils syndicaux et ceux des partis de gauche traditionnels. Dans de nombreux cas, ces mesures suffisent. Mais l'intensité des agressions du système capitaliste à l'égard des peuples les oblige aussi à recourir à divers moyens de sous-traitance de la haine.

L'entreprise est un espace de dictature de plus en plus sévère, y compris dans les pays qui se 
flattent abusivement d'être des Etats de droit comme la France, où l'arbitraire policier se déploie avec une impudence grandissante mais aussi où le code du travail et bien d'autres protections sociales ont été en grande partie démantelés. L'encadrement porte une haine quotidienne à l'égard des salariés sous la forme du harcèlement moral et de la menace du licenciement. Dans cette atmosphère angoissante de concurrence intensifiée entre ceux qui vendent leur force de travail, la haine est portée par bien des salariés à l'encontre des Rmistes, des chômeurs, des jeunes de familles immigrées réputés tous «fainéants, casseurs, dealers », des « femmes nerveuses qui compliquent tout », des « Chinois qui nous piquent notre travail », etc. Les capitalistes font porter au prolétariat la haine d'une partie de lui-même. Au bout du compte chaque prolétaire éprouve, à un certain degré variable bien sûr, une certaine détestation de lui-même. Ce qui s'exprime parfois de façon lapidaire : « Être prolo, c'est la honte. »

A ces représentations démoralisantes et à l'agressivité quotidienne des cadres et des contremaîtres s'ajoute dans un certain nombre de pays le recours à des groupes recrutés dans les milieux populaires, pour casser des grèves, des manifestations, pour assassiner des militants ou pour perpétrer des pogroms contre des immigrés. Les exemples récents sont nombreux. En janvier 2010 ce fut la « chasse à l'homme » contre des immigrés africains en Calabre à Rosarno. En Russie, des bandes de jeunes racistes peuvent agir dans ce sens en toute impunité avec la caution bienveillante de l'oligarchie au pouvoir. En Argentine, il est fréquent que des hommes de main qui sont parfois tout à la fois délégués du syndicat officiel, mafieux et supporters violents d'une équipe de football frappent des grévistes, des étudiants ou des piqueteros. Le 20 octobre 2010 un étudiant de 23 ans, Mariano Ferreyra, qui participait à une manifestation pour bloquer une voie ferrée à Buenos Aires avec des travailleurs licenciés et des militants de son parti, le Partido Obrero, a été tué à la suite d'une fusillade perpétrée par des gens de cette sorte. D'autres du même type ont sévi à nouveau en décembre 2010 au sud de cette ville contre un campement de sans abri pour la plupart d'origine 
bolivienne, paraguayenne ou péruvienne, tuant trois personnes et faisant de nombreux blessés. Le maire de Buenos Aires a confirmé par ses propos xénophobes le fait que cette agression allait dans le sens des intérêts des couches les plus riches qui détiennent les leviers du pouvoir.

\section{Les riches ont la rage}

La lettre de Wall Street de Sylvain Cypel publiée dans Le Monde daté du 29 septembre 2010 avait pour titre : «Finie la peur, les riches ont la rage ». Le correspondant de ce journal évoquait la convergence paradoxale de la colère des classes moyennes aux Etats-Unis contre Barack Obama, perçu comme un suppôt des banquiers de Wall Street et la rage du petit groupe des plus riches contre le même Obama, accusé d'incarner « le socialisme ». Il rapportait les propos de l'économiste Paul Krugman. «La vraie rage politique, celle qui amène des gens à comparer Obama à Hitler ou à l'accuser de trahison, n'allez pas la chercher du côté des Américains qui souffrent réellement. Vous la trouverez plutôt parmi les très privilégiés, ces gens qui n'ont aucune crainte de perdre leur emploi, leur maison ou leur assurance-maladie, mais qui sont littéralement enragés à l'idée de payer des impôts modestement supérieurs ».

Sylvain Cypel donnait ensuite un florilège des considérations émanant de magnats ou de leurs porte-parole dans le Wall Street Journal exprimant une haine délirante contre le caractère « étrange », « étranger», non américain d'Obama, (« The Alien in the White House ») et contre le caractère non américain de la plus timide mesure d' «accompagnement social» de la crise économique.

Il y a là un symptôme extrêmement révélateur car il se situe au niveau de la couche des capitalistes les plus riches et les plus puissants au monde. Ces gens ont surmonté leur peur des conséquences de la crise financière et de la faillite de Lehman Brothers en septembre 2008. A 
présent par tous les moyens nécessaires, ils sont prêts à mobiliser et à développer à leur profit et pour leur perpétuation, les potentiels de haine qui peuvent exister dans tous les pays et dans toutes les classes sociales.

Le prolétariat mondial est constitué de plusieurs centaines de millions d'hommes, de femmes et d'enfants (215 millions d'enfants surexploités selon l'Organisation International du Travail). Il est d'autant plus inquiétant pour les classes dominantes qu'il n'est pas une entité stable, identique à elle-même, dotée d'organisations qu'il suffirait de briser ou de corrompre pour rendre cette masse amorphe et être tranquille.

Sarkozy et son gouvernement en ont fait la désagréable expérience cet automne 2010 avec la mobilisation de trois à quatre millions de salariés qui ont empoisonné leur existence pendant deux mois. En toute logique étatique et bureaucratique, ces salariés et les jeunes qui les ont rejoints n'auraient jamais dû forcer leur talent (?) à ce point et sortir de leur fatalisme. Sarkozy ne pouvait pas prévoir que la visibilité des nantis et de leurs exigences inhumaines est telle qu'elle devient mobilisatrice et pousse à faire de la politique autrement, y compris en dehors des séquences électorales.

La visibilité du prolétariat est faible. On peut aisément le considérer comme une masse amorphe et le faire disparaître de son vocabulaire. Mais il resterait à expliquer comment le capital existe et se reproduit sans prolétaires.

Comme masse chosifiée, comme concept fixe, rigide, il est préférable de s'en débarrasser. De même s'il est pensé de façon creuse comme une classe messianique appelée à sauver le monde grâce aux injonctions éclairées de partis ou organisations d'avant-garde (qui restent d'ailleurs éternellement à construire et pour cause !). Pour autant tous les salariés, petits retraités, chômeurs, sans abris, sans papiers, sans identité nationale bien claire, petits agriculteurs dans la misère ou au bord de la ruine, font bien partie de l'ensemble des prolétaires. Un ensemble constamment 
restructuré, qualitativement et quantitativement, qui se rétracte dans tel secteur ou pays et se développe dans d'autres, qui est détruit ici et surgit ailleurs, au gré des mouvements des capitaux qui s'investissent et se désinvestissent.

Le caractère hétérogène et mobile du prolétariat ne doit pas être nié. D'autant moins qu'il offre des opportunités considérables pour les classes dominantes d'attiser en lui la haine de soi et la haine de l'autre. A l'inverse l'hétérogénéité et la mobilité cassent les routines et multiplient les expériences qui ouvrent des brèches dans l'emprise des dispositifs du capital et éventuellement des voies émancipatrices.

José Chatroussat 